\title{
Clarity of View: An AHP-Based Multi-Factor Evaluation Framework for Driver Awareness Systems in Heavy Vehicles
}

\begin{abstract}
One of the most difficult aspects of any new human-machine interface development is that of evaluating the user's subjective reaction to the system. In some cases, fears about the usability of a new technology may actually prevent its approval for use due to the perception of potential risk. This is especially true in the automotive industry where driver interface design directly affects safety. In this case study, a new evaluation framework is presented: Clarity of View. It incorporates both the results of the industrypublished methods for evaluating a rearview vision system and combine with it additional new factors that contain both subjective perception and quantitative elements.

This AHP based evaluation framework is more closely aligned with the actual decision making process of fleet owners in the trucking industry who are often bombarded with information about potential new safety technology for their fleets, but may otherwise have had a difficult time sorting through the many dissimilar elements of information.

This case study serves as a potential model not only for other driver interface systems within the automotive industry, but for any industry that needs to consider both quantitative measures alongside subjective user perception in order to make a fully informed technology selection decisions.
\end{abstract}

Keywords: automotive, commercial vehicle, user interface design, AHP.

\section{Introduction}

With over 9.2 billion tons of freight moved annually by over 3 million trucks on the highway, the trucking industry is the lifeblood of the US economy [1]. Each year NHTSA reports over 826,000 lane change accidents [3]. Over $25 \%$ of heavy-truck accidents are related to lane-change events. Additionally, driver inattention is the cause of $78 \%$ of crashes across all segments [2]. Several emerging technologies hold great promise to improve situational awareness for the heavy vehicle driver. However, current industry-standard methods do not measure all the comprehensive factors contributing to the overall effectiveness of such systems. This work proposes an improved evaluation framework.

\section{Literature Review}

A literature review is provided in the full paper that summarizes elements of overall system effectiveness, including specific driver awareness technologies; driver awareness human factors; evaluation methods for visibility systems in use today; and the theoretical basis for the application of the AHP methodology for this model [4]. 


\section{Hypotheses/Objectives}

This case study was designed to confirm the usefulness of a multi-factor evaluation framework aimed to expand upon the industry status-quo in use today, thereby enabling a more informed decision-making process.

\section{Research Design/Methodology}

Testing involved the design of a commercial vehicle driving simulator apparatus with 360-degree views to enable the use of rearview mirrors or cameras within a controlled, safe environment. Reaction time and distortion were evaluated within the simulator environment, while a full-sized vehicle was used for static evaluations for the industry standard field-of view measurement, along with glare discomfort. Sixteen test subjects participated in the study, with over half being experienced CDL-licensed drivers. Each factor was evaluated in the method most appropriate for its measurement (direct, pairwise comparison, ranking, or utility curve.) Four sample technology alternatives were evaluated. ExpertChoice software was utilized for the model.

\section{Data/Model Analysis}

The model provided insight into the importance of the multiple factors as compared to the status quo industry standard test procedure that focused on field of view alone. With status quo methods, alternative 4 would have scored highly and likely been chosen for further evaluation via on-road trials. However, by incorporating the additional factors, it could be seen that this technology was not only undesirable, but potentially very unsafe. Alternative B, previously thought to be risky due to perceived distortion, was shown by the model as the best choice overall, with an overall model inconsistency of only 0.04 indicating that judgements were consistent across participants.

\section{Limitations}

This study proved useful for the scenario of comparing a small set of varying alternatives. Difficulty may be seen if attempting to compare too many alternatives or if the alternatives were not distinctly different from one another.

\section{Conclusions}

This new evaluation framework provides a more comprehensive methodology for decision makers in the commercial vehicle industry who must consider both quantitative technical performance data alongside subjective opinion data when making critical safety-related decisions. This model is not proposed for design optimization, but rather as a screening tool to help those responsible for technology selection to identify and focus on the most viable solutions. 
ISAHP Article: A Style Guide for Paper Proposals To Be Submitted to the International Symposium on the Analytic Hierarchy Process 2016, London, U.K.

\section{Key References}

[1] American Trucking Association. "Reports, Trends and Statistics" (2014). www.truckline.com.

[2] Brostrom, Robert, et al. "Correlation between safety assessments in the driver-car interaction design process." Applied Ergonomics. Vol 42 (2011): 575-582.

[3] Pyle, W. "Lane Change Accidents are Caused by Blind Spots and Factors Affecting the Driver's Awareness of Vehicles in Adjacent Lanes of Traffic." Valeo-Raytheon White Paper Series. (2008).

[4] Saaty, Thomas L. "Decision making with the analytic hierarchy process."

International Journal of Services Sciences. 1.1 (2009): 83-98 